\title{
Compact all-fiber plasmonic Airy-like beam generator
}

\author{
Chunying Guan,, ${ }^{1,2,{ }^{*}}$ Ming Ding, ${ }^{2}$ Jinhui Shi, ${ }^{1}$ Pengfei Wang, ${ }^{1,3}$ Ping Hua, ${ }^{2}$ \\ Libo Yuan, ${ }^{1}$ and Gilberto Brambilla ${ }^{2}$ \\ ${ }^{l}$ Key Laboratory of In-Fiber Integrated Optics of Ministry of Education, College of Science, Harbin Engineering University, \\ Harbin 150001, China \\ ${ }^{2}$ Optoelectronics Research Centre, University of Southampton, Southampton SO17 1BJ, UK \\ ${ }^{3}$ Photonics Research Centre, Dublin Institute of Technology, Kevin Street, Dublin 8, Ireland \\ *Corresponding author: cyguan@163.com
}

\begin{abstract}
A compact all-fiber plasmonic Airy-like beam generator is demonstrated. A single slit and a 1D groove array were fabricated by focused ion beam (FIB) milling on the gold deposited end facet of a single mode optical fiber. The single slit excites the surface plasmonic polaritons (SPPs), which are decoupled into free space by the groove array. The phase of decoupling SPPs is adjusted by the grooves position. Experimental generation of the single Airy-like beam has good consistency with theoretical predictions. The transverse acceleration and nondiffraction properties are observed. The interference of double Airy-like beams in the free space is also analyzed. The presented plasmonic Airy-like beam generator is of importance to realize all-fiber optical trapping, beam shaping, and fiber integrated devices.
\end{abstract}

Shaping the phase and amplitude of a light beam is a fascinating topic and provides unique light patterns that play a major role in the field of optical manipulation [1]. Various unusual beams, such as Bessel, LaguerreGaussian, Airy, and collimation beams, have been experimentally obtained in optical micromanipulations and lasers [2-5]. Airy beams have been attracting considerable interest because of their unique properties, including transverse acceleration, non-diffraction, and self-reconstruction [4, 6]. Such beams can persist over very long lengths, allowing the extended guiding of objects using the scattering force. In most experiments, Airy beams were mainly induced by continuous cubic phase patterns generated using a phase-type spatial light modulator (SLM) [7-9]. However, the phase SLM has some intrinsic disadvantages, such as large size, low resolution, and low laser damage threshold. The free space Airy beams can be generated by a combination of positive and negative cylindrical lenses [10]. Surface plasmon polaritons (SPPs) have the capability of spatially confining EM waves to spot sizes considerably smaller than the excitation light wavelength [11]. Plasmonic structures provide compact and integrated optical processing and can obtain specific beam profiles in the near-field or the far-field [5, 12, 13]. Plasmonic Airy beams have been demonstrated experimentally, which were generated on the surface of a metal nanoarray structure [14-15]. In previous investigations, however, most experimental set-ups required extremely precise alignment in the free space or optical Fourier transform [16]. A compact and flexible all-fiber design is more desirable. All-fiber 1D Bessel-like beam [17], superfocusing lens [18], and "rainbow trapping" [19] based the SPPs have been investigated. Recently, an Airy beam generator based on multicore fiber waveguide was also reported [20].

In the present paper, a compact all-fiber plasmonic
Airy-like beam generator is proposed and demonstrated in a conventional single mode fiber. Here, the Airy-like beam generator is composed of a single slit and a 1D groove array on the gold deposited end facet of an optical fiber. Focusing property of double Airy-like beam interference is also investigated.

Figure 1 shows the schematic illustration of the proposed all-fiber plasmonic Airy-like beam generator, similar to the structure used in Ref. 13 on a flat substrate. A gold film was deposited on the end facet of an optical fiber, and a single slit and a $1 \mathrm{D}$ air groove array were fabricated in the gold film. The single slit excites the SPPs, which are then decoupled into free space by the $1 \mathrm{D}$ graded groove array [16]. Here, $h_{0}$ is the thickness of the gold film and also the slit depth, $w_{0}$ is the width of the air slit, $A_{n}$ is the local lattice constant of the nth groove, while $w$ and $h$ represent the width and depth of air grooves, respectively. The relative permittivity $\varepsilon_{g}$ of gold film is $-40.32+2.88$ i calculated by the well-known Drude model [21] at the wavelength $\lambda=980 \mathrm{~nm}$.
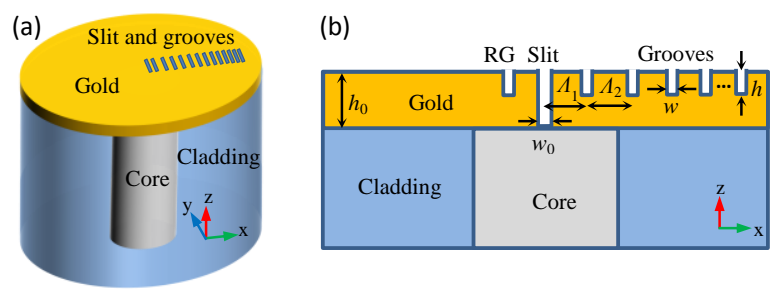

Fig. 1. (a) Schematic illustration of all-fiber Airy-like beam generator. (b) Cross section of proposed device in the x-z plane.

To generate a single Airy-like wave packet, the geometric parameters of the slit and grooves require to be optimized. In order to simplify the calculation, the propagation properties of the proposed device are numerically investigated by the 2D Radio Frequency (RF) module of 
COMSOL Multiphysics 3.5a in the frequency domain. In the simulations, the $980 \mathrm{~nm}$ single mode optical fiber was selected for the discussion. The numerical aperture (NA) of the fiber is 0.14 , the core diameter is $5.2 \mu \mathrm{m}$, the cladding is fused silica and its refractive index was calculated by using the Sellmeier equation. The thickness of the gold film is $200 \mathrm{~nm}$ and the width of the slit is selected as $130 \mathrm{~nm}$. Only the TM-polarized fundamental mode (i.e. with the electric field aligned along $\mathrm{x}$ direction and perpendicular to the long axis of the slit) was launched from the lower input port using boundary mode analysis. The initial phase of decoupled SPPs on the end of fiber, i.e., the phase of the generated Airy beam, is determined by the local position of grating grooves. We know that the recouping waves of -1 order diffraction of two neighbor grooves obey the relation [16]

$$
k_{0} \Lambda_{i} \sin \theta=k_{s p p} \Lambda_{i}+2 \pi
$$

Here $\theta$ is the diffractive angle, $k_{0}$ is the free space wavevector, $k_{s p p}=k_{0} \sqrt{\varepsilon_{d} \varepsilon_{g} /\left(\varepsilon_{d}+\varepsilon_{g}\right)}$ is the wavevector of the SPPs on the metal surface, $\varepsilon_{d}$ and $\varepsilon_{g}$ are the relative permittivity of the air and the gold film. Every neighboring groove has an additional phase difference of $2 \pi$. The phase evolution of the nth groove can be deduced as $\phi_{n}(x)=\phi_{0}+k_{s p p} x+2 n \pi$, where $\phi_{0}$ is the initial phase and $x$ is the distance of the nth groove from the air slit. The asymptotic expression [22] of the field distribution of the finite-energy Airy beam [6] at $\mathrm{z}=0$ can be written as

$$
\operatorname{Airy}(-x, z=0)=\frac{\sin \left(\frac{2}{3}\left(\frac{x}{x_{0}}\right)^{3 / 2}+\frac{\pi}{4}\right)}{\sqrt{\pi}\left(x / x_{0}\right)^{1 / 4}} \exp \left(\frac{a^{3}}{3}-\frac{a x}{x_{0}}\right)
$$

where $x_{0}$ determines the acceleration of the Airy beam and $a$ is the exponential truncation factor. The phase distribution of Airy beam is related to the sine term. The location of every groove on the gold film surface can be calculated by solving the equation [13]:

$\operatorname{Arg}(\operatorname{Airy}(x, z=0))=\frac{2}{3}\left(\frac{x}{x_{0}}\right)^{3 / 2}+\frac{\pi}{4}=\phi_{n}(x)=\phi_{0}+k_{s p p}^{\prime} x+2 n \pi$

$\operatorname{Arg}(\operatorname{Airy}(x, z=0))$ is the phase distribution of Airy beam along the $\mathrm{x}$ direction at $z=0$. The local wavelength $\lambda_{s p p}^{\prime}$ of the SPPs near the slit is slightly smaller than $\lambda_{s p p}$ (967.8nm) due to complex plasmonic distribution [23]. Therefore, the locations of the first few grooves were optimized by using the phase distribution of the SPPs on the gold film surface with the sole air slit (without grooves). The optimized local $\lambda_{s p p}^{\prime}$ results are $955 \mathrm{~nm}$ and $958 \mathrm{~nm}$ for $x<5 \mu \mathrm{m}$ and $5 \mu \mathrm{m}<x<10 \mu \mathrm{m}$, respectively. The local lattice constant at $\lambda_{0}=980 \mathrm{~nm}$ for $x_{0}=1 \mu \mathrm{m}$ and $x_{0}=1.25 \mu \mathrm{m}$ was calculated using Eq. (3) and is presented in Fig. 2, where $\phi_{0} \approx \pi / 5$. The local lattice decreases as the groove distance from the slit increases, except for the first groove.

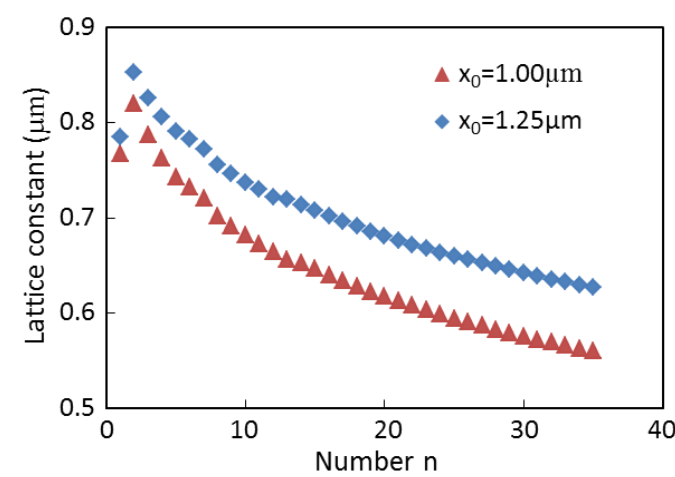

Fig. 2. Calculated local lattice constant of the grooves for $x_{0}=1 \mu \mathrm{m}$ (triangle mark) and $x_{0}=1.25 \mu \mathrm{m}$ (diamond mark).

The amplitude distribution of SPPs along the $\mathrm{x}$ direction in the absence of grooves does not match that of an Airy beam, and its decay is very slow due to small absorption [23, 24]. We can adjust the decay ratio of the SPP amplitude by changing the depth and width of grooves due to scattering and decoupling diffraction, and to match approximately the amplitude of an Airy beam. The deeper the grooves, the faster the amplitude along the $\mathrm{x}$ direction decays. The depth and width of grooves are optimized to $75 \mathrm{~nm}$ and $260 \mathrm{~nm}$ for $x_{0}=1 \mu \mathrm{m}$, and $65 \mathrm{~nm}$ and $250 \mathrm{~nm}$ for $x_{0}=1.25 \mu \mathrm{m}$, respectively. The intensity distributions of the electric field in the $\mathrm{x}-\mathrm{z}$ plane are shown in Fig. 3(a) and 3(b), for a number of grooves equal to 35 . They present the transverse acceleration and nondiffraction characteristic of the Airy beam. The intensity profiles at different $\mathrm{z}$ and the trajectory of the main lobe are described in Fig. 3(c) and 3(d) for $x_{0}=1 \mu \mathrm{m}$. The main lobe is accelerated along the $-x$ direction. The Airylike beam can propagate to about $30 \mu \mathrm{m}$ and $55 \mu \mathrm{m}$ for
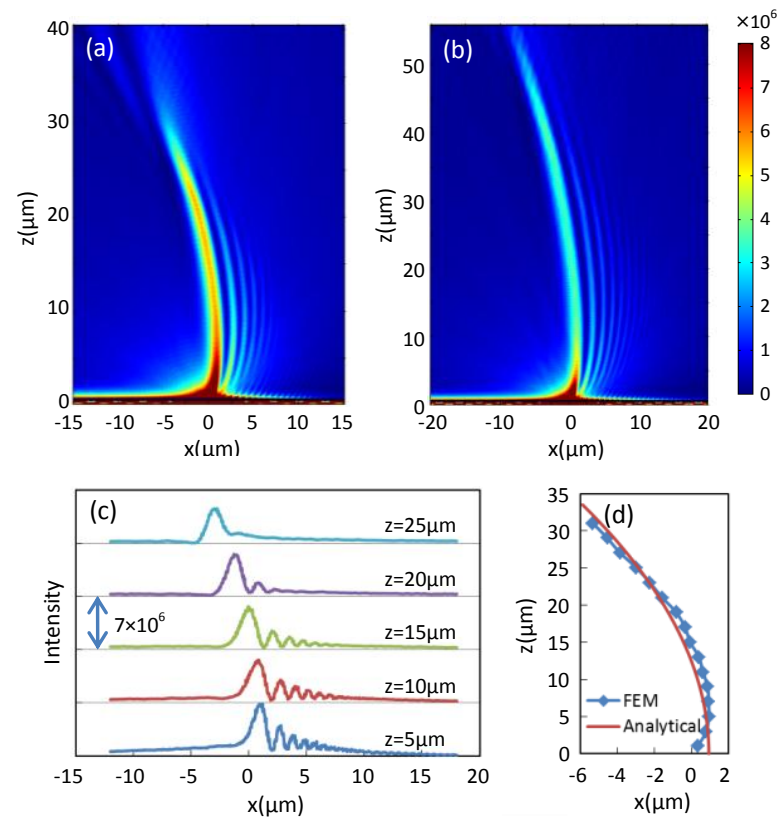

Fig. 3. Calculated intensity distributions of Airy beam generator in $\mathrm{x}^{-} \mathrm{z}$ plane for (a) $x_{0}=1 \mu \mathrm{m}$ and (b) $x_{0}=1.25 \mu \mathrm{m}$. (c) Intensity profiles at different distances $\mathrm{z}$ from the fiber facet. (d) Trajectory of the main lobe beam for $x_{0}=1 \mu \mathrm{m}$. 
$x_{0}=1 \mu \mathrm{m}$ and $x_{0}=1.25 \mu \mathrm{m}$. The acceleration of the former is larger than that of the latter. The analytical trajectory of the main lobe is expressed by $x(z)=z^{2} /\left(4 k^{2} x_{0}{ }^{3}\right)$ and is shown by the red line in Fig. 3(d). In comparison with the ideal finite energy Airy wave, the obtained beam near the fiber facet deflects to the $+x$ direction since the diffractive angle $\theta$ of the decoupling SPP is not zero, thus the decoupling beam is not emitted along the fiber axis. Around $4.1 \%$ of the energy of incident light was converted into the Airy beam propagating in the $\mathrm{z}$ direction, slightly higher than that observed in Ref. [27].

If a reflection groove (RG) is introduced in the left side of the slit to reflect the SPPs propagating along the $-\mathrm{x}$ direction, the efficiency of decoupled SPPs will increase. The optimized distance between the reflection groove and the slit is $0.4 \mu \mathrm{m}$ for $x_{0}=1 \mu \mathrm{m}$ and the corresponding field intensity is presented in Fig. 4(a). Figure 4(b) gives intensity profiles at $\mathrm{z}=10 \mathrm{\mu m}$ for the device without a $\mathrm{RG}$ and with a RG and clearly shows that the reflection groove enhances the main lobe intensity of Airy beam up to $23 \%$ and suppresses the energy of SPPs along the $-\mathrm{x}$ direction.
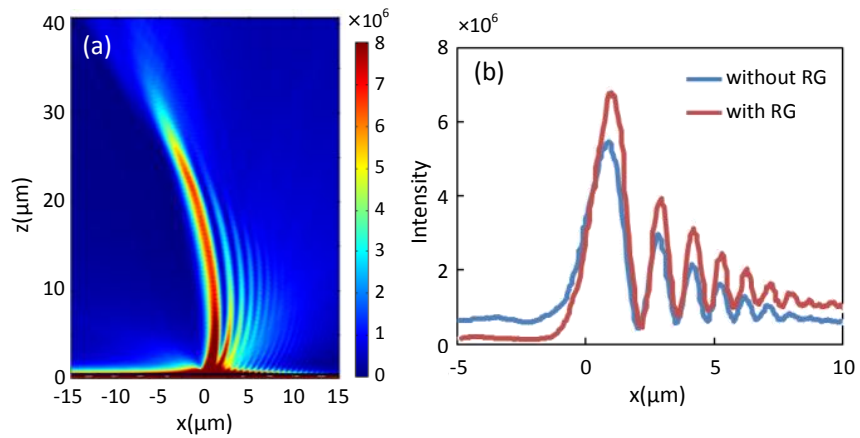

Fig. 4. (a) Intensity of proposed device with a RG ( $\left.x_{0}=1 \mu \mathrm{m}\right)$. (b) Comparison of intensity profiles at $\mathrm{z}=10 \mu \mathrm{m}$ for the device without a RG and with a RG.

In the experiment, a single mode fiber at $980 \mathrm{~nm}$ (Thorlabs, SM980-5.8-125) was coated by a $200 \mathrm{~nm}$ thick gold film thermally deposited on its end facet. A single slit and a 1D graded groove array were fabricated by FIB (Helios 600, FEI Inc.). Figure 5(a) and 5(b) show the scanning electron microscope (SEM) images of a sample with 34 grooves. The widths of slit and grooves are $130 \mathrm{~nm}$ and $260 \mathrm{~nm}$, respectively. The depth of air grooves is estimated to be about $60 \sim 80 \mathrm{~nm}$. The local lattice constant of the grooves is the same as in Fig. 3(a). A $980 \mathrm{~nm} \mathrm{CW}$ laser diode was employed as the incident light. The fiber polarization rotator (FPR, OZ Optics) was used to control the light polarization direction. The transmitted light was then collected by a microscope objective (100×) and beam images were taken by an infrared camera (MicronViewer 7290A, ElectroPhysics). The TM beam images at different distances along the z-axis were obtained by adjusting the distance between the end of a sample and the microscope, and were summarized in Fig. 5(c) and 5(d). The red dashdotted line depicts the trajectory of the beam main lobe. The output beams show a strong similarity with Airy beams. Three side lobes can be clearly observed at $\mathrm{z}=5 \mu \mathrm{m}$. The main lobe is deflected by $4.2 \mu \mathrm{m}$ in the $-\mathrm{x}$ direction at $\mathrm{z}=40 \mu \mathrm{m}$, which is slightly less than expected. The difference between experimental and simulated results is attributed to imperfections in the fabrication process. If the grooves were shallow, the decoupled beams with different emitted directions (different local lattices) would be clearly observed in free space. This can explain the additional lobe observed on the left of the main lobe in Fig. 5(c). Moreover, the lack of perfect uniformity of the grooves depth and width also introduces unwanted scattering, which slightly destroys the desired amplitude distribution.
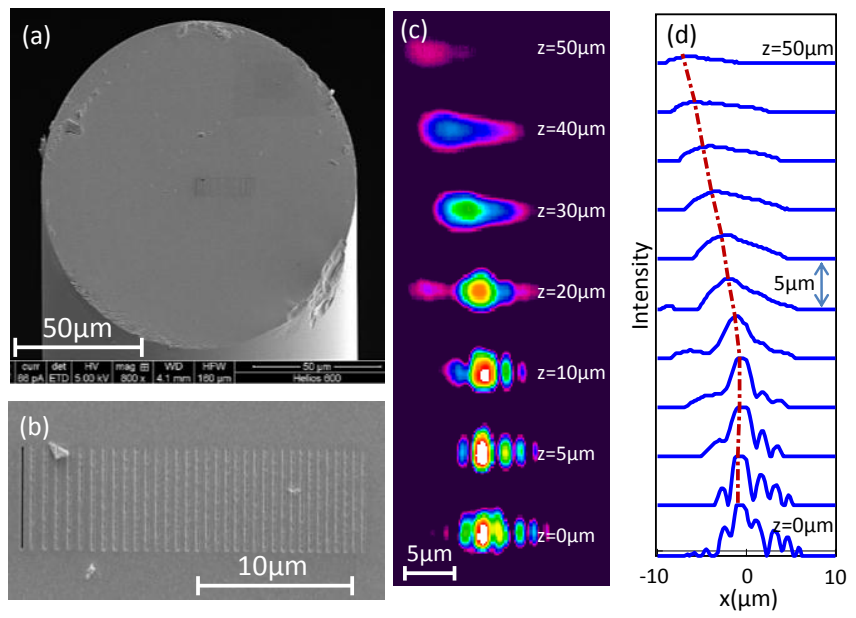

Fig. 5. (a)-(b) Scanning electron microscope (SEM) images of the nanostructured gold coated optical fiber facet. (c) Images and (d) intensity profiles at different distances.

Similarly, a double Airy-like beam generator was designed by arranging two air slits symmetrically in the fiber core area. The distance between the main lobes of two airy beams is limited by the size of the fiber core. The calculated intensities for different distances between two slits are shown in Fig. 6(a), where the RG is absent $\left(x_{0}=1 \mu \mathrm{m}\right)$. The two Airy-like beams interfere in the far field and the two main lobes focus on the axis of symmetry $(\mathrm{x}=0)$, which is analogous to abruptly autofocusing of radially symmetric Airy beams [25]. The intensity profile map for different distances at $\mathrm{x}=0$ (white dashed line in Fig. 6(a)) is also given in Fig. 6(b). The brightest focal spot shifts along the $\mathrm{z}$ axis as the distance between the slits increases. Furthermore, the obvious double-slit plasmonic interference [26] also contributes to the interference field. Therefore, the amplitude of the brightest spot is changed periodically, which is distinct from the interference between perfect two Airy beams [27], and the period is approximately equal to the wavelength of SPP. When the focal spot is brightest the antinodes of the standing-wave of SPP along the interface coincide with the two slits. The electric field intensity on the dashed line in Fig. 6(b) is presented in Fig. 6(c). The brightest focal spot is obtained at $d \approx 2.95 \mu \mathrm{m}$. The spot full-width-at-half-maximum (FWHM) is $0.8 \mu \mathrm{m}$ along a distance $\mathrm{z} \sim 4.9 \mathrm{\mu m}$ and $6.8 \%$ of the input power can be achieved around the bright spot. 

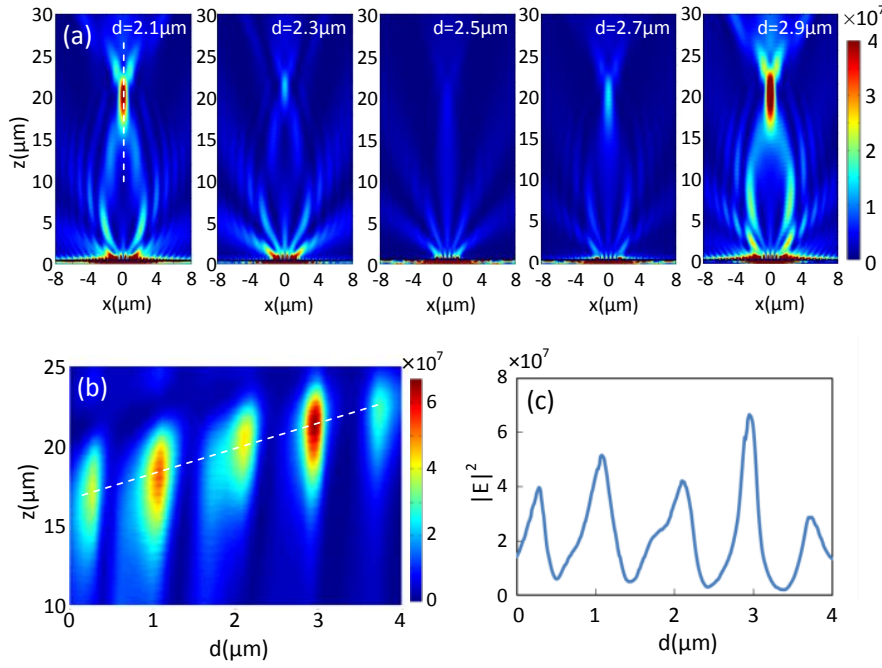

Fig. 6. (a) The calculated interference field of double Airy-like beams generator for different distances between slits $\left(x_{0}=1 \mu \mathrm{m}\right)$.

(b) Intensity profile map for different distances at $\mathrm{x}=0$. (c) Intensity on the dashed of Fig. 6(b).

In summary, a compact all-fiber plasmonic Airy-like beam generator is demonstrated theoretically and experimentally. A single slit and a 1D groove array were fabricated by FIB on the end facet of an optical fiber. The transverse acceleration and nondiffraction property were observed experimentally and show good consistency with the theoretical prediction. The double Airy-like beam interference is also analyzed. All-fiber Airy-like beam generator provides many advantages, such as no need for alignment systems, high flexibility, lower insert loss, and easy portability, which is of importance to realize optical trapping, micromanipulation, beam shaping, and fiber integrated devices.

This work was supported by the National Natural Science Foundation of China (NSFC) under Grant Nos. U1231201, 61275094, 11104043, 61201083 and 11274077 , and in part by the Special Foundation for Harbin Young Scientists under Grant Nos. 2012RFLXG030 and 2013RFQXJ099, and by the 111 project (B13015) to the Harbin Engineering University. P.W. gratefully acknowledges Science Foundation Ireland (SFI) for the International Strategic Cooperation Award Grant No. SFI/13/ISCA/2845. G.B. gratefully acknowledges the Royal Society (London) for his University Research Fellowship.

\section{References}

1. K. Dholakia and T. Čižmár, Nat. Photonics 5, 335 (2011)

2. J. Arlt, V. Garces-Chavez, W. Sibbett, and K. Dholakia, Opt. Commun. 197 (4-6), 239 (2001)

3. P. Prentice, M. MacDonald, T. Frank, A. Cuschier, G. Spalding, W. Sibbett, P. Campbell, and K. Dholakia, Opt. Express 12, 593 (2004)

4. J. Baumgartl, M. Mazilu and K. Dholakia, Nat. Photonics 2, 675 (2008)
5. N. F. Yu, J. Fan, Q. J. Wang, C. Pflügl, L. Diehl, T. Edamura, M. Yamanishi, H. Kan, and F. Capasso, Nat. Photonics 2, 564 (2008)

6. I. M. Besieris and A. M. Shaarawi, Opt. Lett. 32, 2447 (2007)

7. G. A. Siviloglou, J. Broky, A. Dogariu, and D. N. Christodoulides, Phys. Rev. Lett. 99, 213901 (2007)

8. G. A. Siviloglou, J. Broky, A. Dogariu, and D. N.Christodoulides, Opt. Lett. 33, 207 (2008)

9. P. Zhang, J. Prakash, Z. Zhang, M. S. Mills, N. K. Efremidis, D. N. Christodoulides, and Z. Chen, Opt. Lett. 36, 2883 (2011)

10. B. Yalizay, B. Soylu, and S. Akturk, J. Opt. Soc. Am. A 27, 2344 (2010)

11. A. P. Hibbins, B. R. Evans, and J. R. Sambles, Science 308(5722), 670 (2005)

12. N. F. Yu, R. Blanchard, J. Fan, Q. J. Wang, C. Pflügl, L. Diehl, T. Edamura, S. Furuta, M. Yamanishi, H. Kan, and F. Capasso, IEEE Trans. Nanotechnol. 9 (1), 11 (2010)

13. X. M. Tang, L. Li, T. Li, Q. J. Wang, X. J. Zhang, S. N. Zhu, and Y. Y. Zhu, Opt. Lett. 38, 1733 (2013)

14. A. Minovich, A. E. Klein, N. Janunts, T. Pertsch, D. N. Neshev, and Y. S. Kivshar, Phys. Rev. Lett. 107, 116802 (2011)

15. L. Li, T. Li, S. M. Wang, C. Zhang, and S. N. Zhu, Phys. Rev. Lett. 107, 126804 (2011)

16. I. Dolev, I. Epstein, and A. Arie, Phys. Rev. Lett. 109, 203903 (2012).

17. S. Kang, H. Joe, J. Kim, Y. Jeong, B. Min, and K. Oh, Appl. Phys. Lett. 98, 241103 (2011)

18. Y. X. Liu, H. Xu, F. Stief, N. Zhitenev, and M. Yu, Opt. Express 19, 20233 (2011)

19. C. Y. Guan, J. H. Shi, M. Ding, P. F. Wang, P. Hua, L. B. Yuan, and G. Brambilla, Opt. Express 21, 16552 (2013)

20. H. C. Deng and L. B. Yuan, Opt. Lett 38(10), 1645 (2013)

21. M. A. Ordal, L. L. Long, R. J. Bell, S. E. Bell, R. R. Bell, R. W. Alexander Jr., and C. A. Ward, Appl. Opt. 22 (7),1099 (1983)

22. M. V. Berry and N. L. Balazs, Am. J. Phys. 47, 264(1979)

23. P. Lalanne and J. P. Hugonin, Nature Physics 2, 551 (2006)

24. G. Gay, O. Alloschery, B. V. de Lesegno, C. O'Dwyer, J. Weiner, and H. J. Lezec, Nature Physics 2, 262 (2006)

25. D. G. Papazoglou, N. K. Efremidis, D. N. Christodoulides, and S. Tzortzakis, Opt. Lett. 36, 1842 (2011)

26. H. F. Schouten, N. Kuzmin, G. Dubois, T. D. Visser, G. Gbur, P. F. A. Alkemade, H. Blok, G. W. 't Hooft, D. Lenstra, and E. R. Elie, Phys. Rev. Lett. 94 (5), 053901 (2005).

27. A E. Klein, A. Minovich, M. Steinert, N. Janunts, A. Tünnermann, D. N. Neshev, Y. S. Kivshar, and T. Pertsch, Opt. Lett. 37, 3402 (2012). 